\title{
2. Variazione sincronica e mutamento diacronico: il caso di alcuni connettori dell'italiano
}

\author{
Anna Giacalone Ramat \\ Università di Pavia
}

Synchrony and diachrony are two perspectives on the same thing

(Lehmann 2005)

\section{Sincronia e diacronia nella linguistica del Novecento}

Negli studi sulla grammaticalizzazione si assume generalmente che sincronia e diacronia siano strettamente interrelate da molti punti di vista: il mutamento diacronico è promosso e spinto da opzioni che sono presenti in sincronia e a sua volta il mutamento è causa di variazione in sincronia, in un rapporto bidirezionale in cui la variazione è sia l'input che l'output dei processi di mutamento (Giacalone Ramat, Mauri \& Molinelli, 2013). Insomma, come afferma Lehmann (2005), "[s]ynchrony and diachrony are two perspectives on the same thing".

Scopo di questo lavoro è riflettere sulle manifestazioni della relazione tra sincronia e diacronia e sugli strumenti metodologici più appropriati a descriverla prendendo spunto da alcuni casi di mutamento nella storia dell'italiano.

Vogliamo introdurre il tema con alcune considerazioni storiche: se gettiamo uno sguardo all'indietro, possiamo vedere che la stretta relazione tra sincronia e diacronia è in opposizione all'idea di netta separazione delle due prospettive, che è stata dominante nella linguistica del Novecento.

Saussure così definisce gli ambiti di pertinenza della linguistica sincronica e di quella diacronica:

La linguistique synchronique s'occupera des rapports logiques et psychologiques reliant des termes coexistants et formant système, tels qu'ils sont

Come citare questo capitolo:

Giacalone Ramat, Anna, Variazione sincronica e mutamento diacronico: il caso di alcuni connettori dell'italiano. In: Engwall, Gunnel \& Fant, Lars (eds.) Festival Romanistica. Contribuciones lingüísticas - Contributions linguistiques - Contributi linguistici Contribuições linguísticas. Stockholm Studies in Romance Languages. Stockholm: Stockholm University Press. 2015, pp. I3-36. DOI: http://dx.doi.org/I0.I6993/bac.b. License: CC-BY 
aperçus par la même conscience collective. La linguistique diachronique au contraire étudiera les rapports reliant des termes successifs non aperçus par une même conscience collective, et qui se substituent les uns aux autres sans former système entre eux (I955: I40).

Quindi per Saussure anche se nella produzione linguistica dei parlanti si osservano fluttuazioni o mutamenti, essi non hanno alcun valore per la descrizione del sistema (sono aree di imprecisione tollerabili, di variazione libera senza valore). In questa ottica la variazione linguistica viene esclusa dalla teoria linguistica e il mutamento linguistico è inosservabile, almeno nelle sue fasi iniziali.

Lo strutturalismo europeo introducendo la nozione saussuriana di sistema nella linguistica storica ha cercato di superare l'antinomia ricostruendo la diacronia attraverso la somma di sincronie. Gli studi di Martinet (I955, I960) e della sua scuola hanno analizzato il mutamento fonetico e fonologico in relazione agli elementi del sistema nella convinzione che i sistemi tendano a trovare un punto di equilibrio e che il mutamento si attui in punti di squilibrio del sistema. Tuttavia, lo strutturalismo europeo e anche lo strutturalismo americano hanno in genere rivolto scarsa attenzione alla variazione individuale e alle cause del mutamento esterne al sistema; questo atteggiamento è condiviso anche dal generativismo, che ha privilegiato lo studio della competenza su quello dell'esecuzione (salvo alcuni recenti sviluppi nell'ambito della teoria minimalista: van Gelderen 2010).

Altre scuole di linguistica hanno promosso studi sulla variazione nello spazio (geografia linguistica, dialettologia) e nei gruppi sociali (sociolinguistica). Nei modelli sociolinguistici di Labov (I972, I994) il mutamento individuato fin dalle sue prime manifestazioni e seguito nel suo svolgersi è inserito nel contesto sociale. Tuttavia, come osservano Hopper \& Traugott, è mancata un'integrazione dei risultati e metodi delle ricerche sociolinguistiche negli studi sulla grammaticalizzazione (Hopper \& Traugott 2003: 30).

La rigida separazione tra sincronia e diacronia è stata messa in crisi e superata dalla teoria della grammaticalizzazione in cui la stretta connessione tra le due dimensioni è inerente alla nozione stessa di grammaticalizzazione che individua nella variazione sincronica la condizione preliminare per l'attuazione del mutamento.

Fin dagli anni Ottanta i processi di grammaticalizzazione sono stati studiati nel contesto: il modello adottato da Hopper \& Traugott (I993, 2003 ) prevede che nella prima fase del processo di grammaticalizzazione 
i nuovi significati possono sorgere per inferenza pragmatica nel contesto. Questo significa che gli elementi lessicali sviluppano un valore grammaticale solo in certi contesti: la grammaticalizzazione è essenzialmente sintagmatica. Altre affermazioni nello stesso spirito sono che le funzioni grammaticali emergono dall'uso linguistico (Hopper \& Traugott 2003: I7I) e che la teoria della grammaticalizzazione appartiene agli usage-based models.

\section{L'evoluzione della teoria della grammaticalizzazione}

L'interesse principale della prima fase degli studi si è concentrato sulla formazione di elementi morfosintattici come ausiliari, flessioni, marche di tempo e aspetto, che hanno origine da elementi lessicali. L'opera di Lehmann (I982 [1995]) ha segnato il momento di sintesi di questa fase: la proposta di sei parametri per misurare il grado di grammaticalizzazione di un elemento dal punto di vista sincronico e diacronico permette di prevedere che quanto più un elemento è grammaticalizzato, tanto più mostrerà perdita di autonomia sintagmatica e paradigmatica, perdita di libertà di posizione nella frase (fixation), riduzione di portata strutturale (condensation): questi processi, che si attuano gradualmente, hanno come risultato la trasformazione di un elemento lessicale libero, una parola, in un morfema o in un affisso flessivo.

Le proposte di Lehmann hanno ispirato molte ricerche, specialmente a livello morfosintattico: sono stati individuati percorsi graduali che certi elementi lessicali seguono nella produzione di esiti grammaticali: "a noun-to-affix cline”, “a verb-to-affix cline” (Hopper \& Traugott 2003). In questa prospettiva la grammatica è concettualizzata tipicamente come sintassi, morfologia e fonologia, mentre la semantica e la pragmatica rimangono sullo sfondo. Traugott \& Trousdale (20I0: 2 ) notano che " $[t]$ he older view of grammaticalization is the more restrictive one, i.e. that it is a process of reduction, increased dependency and obligatorification".

Ma l'attenzione degli studiosi si è rivolta anche ad altri temi e argomenti, in particolare alla dimensione semantica e pragmatica del mutamento linguistico, che richiedono una concezione più ampia di grammaticalizzazione, in cui alcuni dei parametri di Lehmann possono essere violati (Diewald 20I0). Con la cautela necessaria, poiché il discorso riguarda direzioni di ricerca spesso interdisciplinari entro confini temporali fluidi, possiamo individuare una seconda fase degli studi caratterizzata da "expansion" anziché "reduction", in cui secondo le parole di Croft la grammaticalizzazione è " $\mathrm{t}]$ he process by which grammar is created" 
(Croft 2006: 366). In questa ottica i fenomeni di riduzione ed espansione dipendono dalla funzione grammaticale verso cui un elemento si sta sviluppando e dal tipo di lingua. Funzioni come tempo, aspetto, caso comportano riduzione dello scope e della mobilità posizionale, e aumento della coesione sintagmatica, almeno in lingue flessive. Altre funzioni invece, come la connessione interfrasale, o la marcatura metatestuale possono non richiedere riduzione (Traugott $\&$ Trousdale 2010: 3 ).

Nella seconda fase dei processi di grammaticalizzazione si notano alcune direzioni promettenti che possiamo così riassumere:

a) una spiccata attenzione per i mutamenti semantici per i quali Traugott ha proposto tre tendenze di sviluppo (Traugott \& Dasher 2002), e per la variazione sincronica, lasciando in ombra gli aspetti formali del mutamento (Bisang, Himmelmann \& Wiemer 2004). Però recentemente van Linden, Verstraete $\&$ Davidse (20I0) hanno proposto di redress the balance verso i fattori interni al sistema per render conto dei processi di mutamento.

b) crescente interesse per la dimensione pragmatica del mutamento, per la quale alcuni hanno proposto il termine di pragmaticalizzazione. In questa ottica si segnalano gli studi di Waltereit $(2002,2006)$ su alcuni elementi dell'italiano come guarda, diciamo, la cui funzione è di articolare il discorso e il cui sviluppo si sovrappone in parte ai processi di grammaticalizzazione però non rispetta alcuni parametri di Lehmann.

Di fatto, alcuni casi studiati da Traugott \& Dasher (2002) e da Mauri \& Giacalone (2012) mostrano che elementi come indeed, in fact, instead, o in italiano invece, però, tuttavia hanno origine da costruzioni a testa nominale e hanno seguito nel loro sviluppo storico i percorsi tipici della grammaticalizzazione, come perdita di proprietà categoriali, espansione dei contesti d'uso, e sono entrati nella dimensione pragmatica nel momento in cui hanno sviluppato valori intersoggettivi o valori procedurali, cioè di articolazione del testo dando luogo a polisemie varie. Giustamente Traugott insiste nel sostenere che questi sono veri casi di grammaticalizzazione "which connect single utterances of longer discourse sequences establishing anaphoric and cataphoric relations and imposing a hierarchy to the sequence" (Traugott 2003: 643). Il termine pragmaticalizzazione allude ad una visione più ristretta dei fenomeni pertinenti al campo della grammaticalizzazione che considera i parametri di Lehmann come caratteristiche ineludibili del processo. In realtà la pragmaticalizzazione può essere vista come un caso speciale di grammaticalizzazione, in cui alcuni elementi linguistici che avevano già subito un processo di grammaticalizzazione, hanno fatto un passo 
ulteriore verso il dominio della pragmatica e sono stati recrutati per servire funzioni discorsive.

Un caso chiaro di questo processo in due fasi è dato da instead e dal suo corrispondente italiano invece, che mostrano un classico percorso di grammaticalizzazione seguito da una estensione pragmatica. Una costruzione con testa nominale stede "luogo" seguita da un complemento preposizionale ha subito un processo di decategorizzazione del nome (che non è più modificabile), di univerbizzazione e anche di estensione della host class (è stato usato per designare anche relazioni astratte). In seguito instead ha sviluppato un valore di connettivo per esprimere principalmente la relazione di sostituzione (instead of... + gerundio) (scope increase). Questa relazione implica il riferimento a luoghi astratti, "in the mental world of values and functions” (Traugott \& Dasher 2002: 636). Gli usi di instead come marca discorsiva sono stati analizzati da Fraser (2007).

Invece sostanzialmente ha seguito un percorso analogo: l'origine è dal latino vece $(m)$ (rimasto in italiano moderno in espressioni fisse: in vece sua, fare le veci di) che, passato attraverso decategorizzazione e univerbizzazione, ha dato luogo a usi di preposizione, di avverbio contrastivo e di congiunzione subordinante seguita dall'infinito:

(I) porta gli occhiali, invece ci vede benissimo

(2) invece di studiare è uscito a fare una passeggiata

Infine invece può essere usato come marca discorsiva di apertura di turno, in cui il valore contrastivo è molto attenuato:

(3) sì ecco io vorrei invece fare un'altra domanda... (LIP, FC5 86)

c) Le nozioni di soggettivizzazione e di costruzionalizzazione hanno goduto di crescente popolarità nelle ricerche linguistiche (Davidse, Vandelanotte \& Cuyckens 20 Io, Traugott \& Trousdale 20 Io, Trousdale 20I3). Com'è noto, i processi di soggettivizzazione e di intersoggettivizzazione riguardano mutamenti semantici che mettono in primo piano gli atteggiamenti ed opinioni del parlante e la sua attenzione verso l'ascoltatore: questi processi si sono rivelati un fattore motivante cruciale per il processo di grammaticalizzazione. Le ricerche si sono concentrate finora su alcune aree della grammatica, gli avverbi, i modali, le particelle discorsive, le particelle scalari e le congiunzioni di subordinazione come ingl. while o ital. mentre.

Gli approcci costruzionisti, elaborati per la descrizione sincronica (Goldberg I995), sono stati con profitto applicati alla diacronia e agli 
studi di grammaticalizzazione (Bergs \& Diewald 2008). La cornice teorica della Construction Grammar offre alcuni vantaggi per le indagini sul mutamento linguistico: anzitutto l'attenzione ai tratti contestuali e la focalizzazione sul momento iniziale del mutamento e anche la mancanza di una divisione netta tra semantica e pragmatica. Questi aspetti rendono la grammatica costruzionista particolarmente adatta a indagare l'interfaccia sincronia/diacronia e la nascita del mutamento dalla variazione. Anche la maggiore disponibilità negli anni recenti di corpora di lingua parlata e scritta facilita il compito di indagare la nascita di nuove costruzioni e di verificarne le frequenze delle varianti in gioco in un dato contesto di mutamento. Tra gli esempi più noti di analisi costruzioniste menzioniamo Traugott (2008) sulla grammaticalizzazione di costruzioni partitive del tipo NPIof $\mathrm{NP}_{2}$ (a bit of an apple) a determinanti complessi o quantificatori (a bit of a liar); altre indagini hanno studiato elementi strutturalmente simili a questi ultimi, ma con funzioni diverse: sort of, kind of (Denison 2005, Mihatsch 2007 e Margerie 2010).

La cornice teorica costruzionista è adottata negli studi di caso che costituiscono la seconda parte di questo lavoro.

d) Gli studi sincronici e diacronici sulla natura graduale o istantanea (abrupt) del mutamento linguistico e sulla nozione di gradience intesa come variazione nell'appartenenza a categorie linguistiche (Traugott $\&$ Trousdale 20I0) hanno promosso un vivace dibattito. Come esempio di gradience tra nomi o aggettivi che sono vicini al prototipo della categoria Nome, Aggettivo ed altri membri marginali che mostrano restrizioni distribuzionali si può considerare il caso di utter. Utter non è modificabile da quantificatori e non ha uso predicativo: an utter disgrace, *very utter, "the problem is utter, quindi è meno prototipico di un aggettivo come thin (Aarts 2007: I06). Se tuttavia, seguendo l'approccio sincronico di Aarts (2007: 98-IOI), consideriamo la gradience all'interno di una classe di verbi (ad es. da verbo principale hope to, a semi-ausiliare have to, a modale marginale dare, a modali centrali can), ci rendiamo conto che la gradience può essere letta come un percorso diacronico che mostra il graduale costituirsi della classe dei modali: gradience e gradualness sono in stretta relazione, non si escludono reciprocamente, ma sono complementari.

Secondo Traugott \& Trousdale (20I0) e Trousdale (in stampa) il mutamento linguistico è fatto di micro-steps, di per sé istantanei, che però danno l'apparenza della gradualità. Questa interpretazione contribuisce a mettere la relazione tra la variazione sincronica e il mutamento diacronico al centro del dibattito. 


\section{Alcuni case studies: origine e sviluppo dei connettori contrastivi in italiano}

Le analisi qui presentate sono basate sull'esame qualitativo e quantitativo dello sviluppo di alcuni connettori italiani e intendono identificare i ruoli della frequenza, della sintassi e del contesto nel percorso di grammaticalizzazione (Mauri \& Giacalone Ramat 20I2). Sono stati presi in esame però, tuttavia e mentre dalla documentazione più antica fino all'italiano moderno, con l'ausilio dei corpora dell'OVI, della Biblioteca Italiana, della LIZ, e del LIP.

I connettivi avversativi codificano un contrasto semantico tra due proposizioni. Si tratta di una nozione astratta di carattere soggettivo che deriva da altre nozioni, più concrete, temporali o causali (Traugott \& König I99I). Nelle lingue romanze si osservano percorsi di sviluppo simili dal significato originario (source meaning) a quello contrastivo (Giacalone Ramat \& Mauri 20I2):

- causa > contrasto (it. però, sp. pero, fr. pourtant)

- simultaneità > contrasto (it. mentre, sp. mientras, ant.franc. domientres)

- continuità > contrasto (it. tuttavia, fr. toutefois)

Il parametro semantico centrale dell'analisi è stata la compatibilità o incompatibilità tra il significato originario e il significato contrastivo. Abbiamo cercato di valutare per ciascuna occorrenza se essa era compatibile col significato originario di ciascun elemento (source meaning) o con quello di arrivo (target meaning), ossia il valore contrastivo. Una terza possibilità ammessa dall'analisi è la doppia compatibilità che si trova nei contesti che ammettono sia il valore originario temporale o causale sia quello avversativo (dual compatibility), da alcuni chiamati contesti ambigui o critical contexts (Diewald 2002) o bridging contexts (Heine 2002). Abbiamo evitato il termine ambiguità perché non ci è sembrato sufficientemente esplicito sull'origine e le cause del fenomeno. Si potrebbe pensare che l'ambiguità nasca nel discorso del parlante, nel senso che il parlante stesso potrebbe essere incerto sul valore da dare a una data occorrenza di un connettore nel suo discorso; tuttavia di solito i parlanti non scelgono di proposito di essere ambigui. Sembrerebbe più plausibile pensare che sia l'ascoltatore che interpreta con l'aiuto del contesto il messaggio e può aggiungere, se il contesto lo permette, un'inferenza contrastiva al messaggio, 
oltre alla relazione temporale o causale espressa dal connettore. Il termine ambiguità implica mancanza di chiarezza, mentre in realtà si tratta della possibilità di una lettura multipla, che è poi il locus del mutamento.

Ci sono dei criteri per valutare la compatibilità: ad es. se troviamo un uso di mentre che collega due proposizioni chiaramente collocate in momenti temporali diversi, possiamo sicuramente parlare di incompatibilità col significato originario: oggi è piovuto a lungo, mentre la settimana scorsa abbiamo avuto un tempo magnifico.

Seguendo la tradizione degli studi sui percorsi di grammaticalizzazione (Traugott 2008, Diewald 2002, 2010), abbiamo individuato nella nostra analisi delle fasi o stadi del processo, ciascuno caratterizzato $\mathrm{da}$ un insieme di proprietà semantiche e sintattiche dei contesti in cui le forme in questione occorrono. La nostra analisi rientra nell'insieme degli approcci costruzionisti al mutamento linguistico.

Abbiamo preso in considerazione la frequenza relativa con cui certe proprietà semantiche e morfosintattiche occorrono in contesti specifici per individuare le associazioni preferenziali tra certi tratti e certi contesti. Come sostiene Bybee $(2006,2007)$, la frequenza è un fattore cruciale nel momento in cui i parlanti attuano una rianalisi della forma e funzione di un elemento dal valore originario a quello di arrivo. In tale momento si osserva un aumento significativo della frequenza di contesti a doppia compatibilità che sono quelli che ammettono la reinterpretazione e rendono possibile il mutamento semantico. Tali contesti infatti devono essere sufficientemente frequenti per permettere ai parlanti la reinterpretazione della costruzione come unità associata sistematicamente al nuovo significato.

\subsection{Il caso di però}

Nell'italiano moderno però è un connettore contrastivo con valore controaspettativo (Mauri \& Giacalone Ramat 20I2) usato per codificare il contrasto che nasce da un'aspettativa negata (cfr. il tedesco aber) (Scorretti I988: 230-23I), ossia la negazione dell'aspettativa dichiarata nella prima proposizione.

(4) Mario gioca bene però perde in continuazione.

Inoltre nell'italiano moderno non è difficile trovare usi in cui però ha funzione discorsiva, serve cioè a introdurre in una conversazione l'opinione dell'interlocutore che oppone il suo punto di vista a quanto detto 
dall'altro interlocutore. Ecco alcuni esempi tratti dal LIP (per esempi di italiano scritto si veda Giacalone Ramat \& Camugli 20II):

(5) C: cioè l'ho fatto io questo qui me lo posso rifare

A: ah si però tu l'hai fatto a macchina o con il computer

C: no al computer

A: ah che amore vallo a ristampare con l'interlinea due

(un colloquio tra giornalisti) (LIP, FA $422 \mathrm{~A}$ )

(6) ammazza la vecchia col cric è troppo bellina

C: sì ma quella non è inventata è vecchia come il cucco però

(alla ricerca di motivi musicali) (LIP, FA2)

Ma in origine però aveva valore causale: lo sviluppo diacronico da connettivo causale-resultativo a connettivo contrastivo si può ricostruire secondo il seguente percorso a stadi (Giacalone Ramat \& Mauri 2008):

I. stadio: dal latino per hoc "per questo motivo" all'italiano antico però "perciò" con valore risultativo, accanto a però che = "perché", causale:

(7) Ed elli a me: «Però che tu trascorril per le tenebre troppo da la lungi,/ avvien che poi nel maginare abborri./ Tu vedrai ben, se tu là ti congiungi,/ quanto 'l senso s'inganna di lontano;/ però alquanto più te stesso pungi».

(Dante Alighieri, Inferno XXXI, 22-27)

(Virgilio rimprovera Dante che da lontano aveva creduto di vedere delle torri, mentre si trattava di giganti: poiché tu trascorri con lo sguardo troppo lontano, accade che nel rappresentarti alla mente l'oggetto della tua visione ti allontani dal vero (abborri).

\section{2. stadio: non però}

I contesti per la reinterpretazione (o form-function reanalysis) di però in senso contrastivo sono quelli negativi. Già nel XIV sec. troviamo contesti ambigui che ammettono due interpretazioni:

(8) Poi giunse: "Figlio, queste son le chiosel di quel che ti fu detto; ecco le 'nsidiel che dietro a pochi giri son nascose./ Non vo' però ch' a' tuoi vicini invidie, $[\ldots]$...

(Dante Alighieri, Paradiso XVII, 94-97, canto di Cacciaguida)

Si tratta di un contesto ambiguo che ammette tanto la lettura risultativa "perciò" quanto quella controaspettativa "però, tuttavia" : la 
negazione ha scope su però, ma anche sulla relazione interfrasale che però codifica e quindi nega la sequenza causale tra gli states of affairs connessi, ossia nega l'aspettativa creata dalla prima frase. Pertanto nei contesti in cui però è nello scope della negazione si genera un'inferenza contrastiva che favorisce la rianalisi di però come la marca esplicita del contrasto. I contesti a doppia compatibilità aumentano di frequenza durante i secoli XIV e XV (Mauri \& Giacalone Ramat 20 I 2 per dettagli) ed è in questo periodo che è plausibilmente avvenuta la rianalisi di però da resultativo a contrastivo.

3. stadio: specializzazione sintattica.

Emerge nei secoli XVI e XVII una distribuzione sintattica complementare che è associata sistematicamente al valore originario o al valore avversativo di però, mentre i contesti a doppia compatibilità diminuiscono:

- (e) però in posizione iniziale ha valore resultativo;

- non (VP) però e ma però in posizione posposta hanno valore avversativo.

Solo dall'inizio del XVII sec. si trovano casi in cui la negazione può essere tralasciata perché ormai però ha assunto valore avversativo.

(9) La guerra e queste querele posero in silenzio per questo anno le trattazioni di concilio; le quali però ritornarono in campo il seguente 1544.

(Paolo Sarpi, Istoria del Concilio Tridentino I, I6I9)

4. stadio. Estensione di però avversativo a qualsiasi posizione e scomparsa del valore resultativo: questo sviluppo si attua nel corso del XIX secolo.

(г) - Non per sempre, però - sibila

(Sandro Veronesi, Caos calmo, p. I75)

\subsection{Il caso di tuttavia}

Il valore originario di tuttavia < latino tota via, era temporale "sempre, continuamente", mentre nell'italiano moderno tuttavia esprime il contrasto controaspettativo. Per questo percorso di mutamento i parametri considerati sono stati la posizione all'inizio della proposizione, all'interno, o dopo il verbo e la co-occorrenza con ma o con una proposizione concessiva precedente (Giacalone Ramat \& Mauri 2009). 
I. stadio: tuttavia avverbio temporale di predicato "sempre, continuamente"

(I I) Fortemente mi 'navanzal e cresce tuttavial lo meo innamoramento

(Rinaldo d'Aquino, sec. XIII)

2. stadio: alcuni contesti compatibili sia col valore originario sia col valore contrastivo si trovano già fin dal XIII sec. Quando tuttavia è in posizione iniziale può essere interpretato come riferito alla continuità temporale della proposizione che segue tuttavia, oppure come riferito ad una nozione più astratta di continuità che coinvolge entrambe le proposizioni: [clause $a$ ] tuttavia [clause $b$ ]. In questa seconda interpretazione anaforica il contrasto può sorgere nel caso che la [clause $a$ ] sia in conflitto con la [clause $b$ ].

(I2) E avegna che lla naturale memoria sia perfettissima cosa a l'uomo, tuttavia è molto debole e fragile [...]

(Bono Giamboni, Fiore di Rettorica [58], I292)

Questo passo ammette sia l'interpretazione temporale "sempre", sia quella contrastiva "nonostante". Diversamente da però i contesti a doppia compatibilità raggiungono il picco più alto già nel XIV sec., il che mostra che il mutamento è iniziato precocemente, forse prima della documentazione scritta.

(13) Non ha dubbio che questo che voi dite ha assai dell'apparente; tuttavia potete vedere come la sensata esperienza mostra il contrario

(Galileo Galilei, Dialogo sopra i due massimi sistemi del mondo, Giornata Prima, I624-1630)

Questo contesto, più tardo, non è compatibile col valore temporale originario e può essere interpretato solo come contrastivo.

3. stadio. Specializzazione sintattica: dal XVI sec. la distribuzione di tuttavia sembra osservare una tendenza alla "specializzazione sintattica": in posizione iniziale prevale il valore contrastivo, in posizione postverbale per lo più il valore temporale. Si noti che il valore temporale si riscontra ancora nel XIX sec.

4. stadio. Nell'italiano contemporaneo tuttavia ha solo valore controaspettativo e piena mobilità sintattica all'interno della proposizione.

(I4) naturalmente la cosa non ebbe seguito ma fu tuttavia un atto che poteva chiarire alcuni sospetti [...] 


\subsection{Dalla simultaneità al contrasto nella diacronia di mentre}

Il tipo di contrasto codificato da mentre non implica la negazione di un'aspettativa, come nel caso di però e tuttavia, ma semplicemente un'opposizione tra due proposizioni caratterizzate da qualche tratto antonimico percepito dal parlante e/o dall'ascoltatore.

Mentre, dal latino dum+interim, ha corrispondenze nel mondo romanzo: ant.franc. domientres, ant.ital. domentre, dementre, ant. sp. domientre.

I. stadio: mentre nell'italiano del XII e XIII secolo (spesso associato che: mentre che) esprime la simultaneità, la co-estensione "finché, per tutto il tempo che" e anche la relazione di terminus ad quem "finché non".

(15) Ella non temè niente la forza di Oloferne, anzi si mise a rischio di morte, per scampare lo popolo, e si l'uccise mentre ch'egli dormia

(Tesoro volg. [ed. Gaiter], XIII ex. (fior.)> [L. I, chap. 59| p.I40])

(16) [...] Agata disse: "Mentre ch'io viverò, sempre il chiamerò con la lingua e col cuore".

(Leggenda Aurea, XIV sm. (fior.) [chap. 39, S. Agata | p. 340])

(I7) sappi che se' nel secondo girone [...] e sarai mentrel che tu verrai ne l'orribil sabbione.

(Dante Alighieri, Inferno XIII, I7-I9 “finché non”)

I valori co-estensivi non sono continuati nell'italiano moderno.

\section{2. stadio}

I contesti in cui mentre ha valore di sovrapposizione temporale possono contenere elementi antonimici che possono suggerire un'opposizione perché due situazioni simultanee si prestano ad essere confrontate sulla base delle loro differenze (oltre o piuttosto che delle somiglianze). Uno degli esempi più antichi è il seguente, es. (I 8), in cui le due situazioni "pensare di fare il furto" e "vangare" si sovrappongono temporalmente, ma denotano un'opposi-zione nelle intenzioni soggettive. Casi del genere fanno parte dei contesti critici, in cui al valore di simultaneità si associa un'opposizione semantica polare, un'opposizione astratta:

(18) Costui sì lavorava uno orto; sì che alcuni, volendoli torre de l'erbe del suo orto, mentre che pensavano di fare il furto per tutta la notte, diligentemente vangavano l'orto [...]

(Leggenda Aurea, XIV sm. (fior.) [cap. I9, S. Felice |p. 204]) 
Nell'es. (I9) la simultaneità temporale è in secondo piano e l'opposizione polare è stabilita a un livello astratto e soggettivo:

(19) [...] sciocchezza grande è il voler noi terreni esser arbitri delle grandezze, e regolatori delle loro locali disposizioni, mentre siamo ignorantissimi di tutti i loro affari e interessi

(Galileo Galilei, Dialogo sopra i due massimi sistemi del mondo, Giornata III, I624-I630)

I contesti con doppia compatibilità sono piuttosto rari nei secoli XIV e XV, mentre nel corso del XVII sec. diventano molto più frequenti (Mauri \& Giacalone Ramat 20 2 per i dati sulle frequenze).

\section{3. stadio}

In questo stadio appaiono dei contesti isolanti, in cui l'interpretazione di simultaneità è esclusa: si tratta di solito di contesti in cui è presente un'indicazione esplicita di distanza temporale o che riportano eventi non fattuali non collocabili nel tempo:

(20) Le famiglie dei patrioti si vollero esentare, mentre forse era più giusto che dassero le prime l'esempio di contribuire con generosità ai bisogni della patria.

(Vincenzo Cuoco, Saggio storico sulla rivoluzione napoletana del I799 - XXVIII Imposizioni)

\section{4. stadio}

Mentre nell'italiano contemporaneo ammette la coesistenza del valore di simultaneità e di opposizione ("layering”, Hopper \& Traugott 2003: I24 sgg.). Lo statuto sintattico di mentre richiede qualche riflessione: di solito viene considerato una congiunzione subordinante (Serianni I988), tuttavia il mentre non temporale sembra avere proprietà di congiunzione di coordinazione. Per accertare se la proposizione introdotta da mentre ha uno statuto coordinato o subordinato rispetto all'altra proposizione, si possono applicare $\mathrm{i}$ test di assertività come la tag question (vero?) e la negazione di frase (clausal negation: non è vero che) che sono usati nella linguistica tipologica per distinguere coordinazione e subordinazione (Mauri 2008, Cristofaro 2005). Nel caso dell'esempio (2 I) soltanto la proposizione principale viene negata; questo significa che la proposizione introdotta da mentre non ha forza illocutiva propria ed è subordinata; nel caso invece di (22) (esempio da Scorretti I988: 238) entrambe le proposizioni rispondono ai test di assertività, quindi 
entrambe hanno forza illocutiva. Il rapporto che le unisce è quindi di coordinazione.

(2I) Mentre parte, dal finestrino ti fa "io mi fermo a Mestre" Non è vero che, mentre parte, [dal finestrino ti fa "io mi fermo a Mestre"]

(22) Gino raccoglie francobolli mentre Vincenzo fa collezione di ombrelli colorati

Non è vero che [Gino raccoglie francobolli mentre Vincenzo fa collezione di ombrelli colorati]

Occorrono studi più approfonditi per valutare il mutamento sintattico di mentre da subordinatore a coordinatore. Si può far notare che la possibilità di anteporre tutta la frase introdotta da mentre (Mentre Vincenzo fa collezione di ombrelli colorati, Gino raccoglie francobolli) è un tratto tipico della relazione di subordinazione impossibile per la coordinazione. Comunque non abbiamo prove che un eventuale mutamento sintattico abbia influenzato lo sviluppo della funzione avversativa. Basti qui ricordare che, come osserva Kortmann (I997: 56), molti subordinatori avverbiali possono assumere funzioni di coordinazione: il ted. weil "perché" può essere usato per asserire le ragioni di una proposizione precedente, $\mathrm{e}$ in tal caso non è più un subordinatore causale.

\section{La grammatica costruzionista diacronica e le costruzioni emergenti}

Ricordando con Hopper (I987) che la grammatica non è un'entità statica, ma un sistema dinamico in movimento, discuteremo alcuni casi di emergent grammar nell'italiano contemporaneo, ossia di costruzioni grammaticali in via di sviluppo, che non compaiono ancora nelle grammatiche e nei dizionari e non fanno parte dell'uso comune. Questi casi sono interessanti non solo perché mostrano l'italiano in movimento, ma anche perché offrono un testing ground per le teorie sul mutamento linguistico di cui abbiamo discusso sopra, in particolare sulla gradualità e sulla variazione sincronica. È possibile controllare su corpora estesi l'espansione del mutamento e la sua frequenza. Dai due studi recenti di Voghera e di Giacalone Ramat e Mauri, che presentiamo qui sotto, sembrano emergere conferme delle analisi metodologiche proposte e applicate a dati storici negli studi sulla grammaticalizzazione. 
Miriam Voghera (20I2 e in stampa) ha esaminato gli usi non nominali di tipo nell'italiano contemporaneo. Si tratta di sviluppi recenti che hanno dato origine a nuovi significati e nuove funzioni grammaticali, la cui distribuzione varia in relazione a dimensioni diverse, testuali e di registro (quindi variazione sincronica). Si sono venute a creare nuove unità di forma e significato per esprimere nozioni di vaghezza e approssimazione. Si noti inoltre che la vicenda di tipo si confronta con quella di termini simili in lingue europee, che hanno conosciuto analoghi percorsi di mutamento: inglese type, kind of, sort of, like, svedese typ, francese genre (Denison 2005, Mihatsch 2007, Margerie 2010).

A partire dal nome tipo "modello", attestato dal XVI secolo, Voghera ricostruisce un percorso di grammaticalizzazione e di decategorizzazione, che individua nel sintagma preposizione del tipo di/sul tipo di i "bridging contexts" o contesti critici (Diewald 2002) verso usi non nominali con caduta delle preposizioni. La funzione di queste costruzioni è di attribuire una qualità o una caratteristica a un SN attraverso una similitudine o analogia con un altro elemento o insieme di elementi:

(23) Si istituiranno scuole speciali sul tipo della scuola all'aperto [...]

(Regolamento per difesa contro le malattie infettive nelle scuole, ottobre I92 I)

(24) la guerra divenne una guerra di civiltà, una guerra tipo «Crociate» (A. Gramsci, Quaderni del carcere, I937)

Il complesso sviluppo diacronico di tipo, molto recente, si svolge secondo una successione di passi discreti e definiti (e senza cancellare le fasi precedenti):

nome $>$ preposizione $>$ avverbio $>$ avverbio di frase $>$ marca discorsiva

Alcuni esempi di usi non nominali di tipo:

tipo come preposizione, con funzione di identificazione approssimativa:

(25) La trattava tipo segretaria insomma (LIP-Conversazione)

tipo come avverbio "approssimativamente, più o meno": il parlante sceglie di lasciare la scelta non determinata:

(26) Partiamo domani // tipo// 
tipo come discourse marker, con funzione di approximator, per attenuare la forza pragmatica di un'espressione o come focalizzatore non contrastivo (come like in inglese):

(27) alla fine gli lascio un messaggio proprio tipo a mezzanotte (Chatline)

(28) ma tipo se faccio un caffè? (Conversazione - LIP)

(29) se uno dei due tipo al cambio dell'ora scende e mette il nome? (conversazione)

Secondo Voghera la distribuzione degli usi non nominali di tipo varia in relazione alle dimensioni testuali e di registro: la frequenza è maggiore nel parlato, specialmente nei dialoghi, in cui le condizioni comunicative favoriscono l'uso di espressioni vaghe (vague language: Channel I994). L'analisi, di tipo qualitativo piuttosto che quantitativo, è un buon esempio di come sincronia e diacronia giochino un ruolo cruciale l'una nella spiegazione dell'altra.

Caterina Mauri e Anna Giacalone Ramat (20I I) hanno studiato un uso specifico dell'italiano piuttosto che, un caso che le due studiose hanno chiamato di indefinitezza restrittiva (restricted indefiniteness). Si tratta di una costruzione emergente col significato di "e qualcosa del genere" caratterizzata dalla struttura $[X P /$ clause piuttosto che $]$ in posizione finale di proposizione, dove XP sta per una qualsiasi categoria maggiore SN, SP, SA, SV: ${ }^{2}$

(30) [...] spesso lo metto anch'io [in zaini piuttosto che], ma una protezione in più non fa mai male [...] (forum discussion)

Piuttosto (che) è in primo luogo un comparativo preferenziale (questo è il significato segnalato nelle grammatiche: Serianni I989):

(3I) preferiscono mangiare piuttosto che essere mangiati (COLFIS corpus)

o un comparativo preferenziale con valore scalare, come in (32), in cui piuttosto che introduce l'alternativa meno desiderabile (equivalente a anziché, invece di).

(32) piuttosto che stare con uno che ha la fissa per il calcio, rimango sola [...] (http://forum.alfemminile.com/)

Piuttosto che può essere anche usato nel parlato, meno frequentemente nello scritto, come congiunzione disgiuntiva con valore indefinito:

(33) [...] una serie di attività 'che le persone che lavorano non [...] possono sviluppare quindi non so dall'andare a fare le file alla posta eh 
piuttosto che avere una baby-sitter all'ultimo momento [...] piuttosto che non so organizzare ecco una festa per una mamma che lavora al suo bambino' [...] (LIP, R E 8 I2 B)

In questo caso piuttosto che è posto tra alternative simmetriche e non preferenziali e si sovrappone in parte con $o$, ma presenta una serie di restrizioni: ad es. può comparire solo in frasi dichiarative, non in interrogative; inoltre la lista delle alternative potenziali non è esaustiva e non è mirata ad alcuna scelta (Bazzanella \& Cristofoli I988, Brucale 20I2).

Infine piuttosto che compare con una funzione indefinita restrittiva, come nell'es. (29) e nell'es. (34): si tratta di una costruzione presente nell'italiano parlato, specialmente settentrionale, che non è stata finora oggetto di studio:

(34) Spesso c'è il problema di dire "dove si va", magari per un giro pomeridiano, piuttosto che. E magari dietro casa si hanno itinerari che non si conosce minimamente semplicemente perchè "non ho mai girato a destra”. (forum discussion)

In questo caso piuttosto che può essere parafrasato con "o qualcosa del genere" e denota una serie di alternative alla proposizione che precede che il parlante non specifica o non vuole specificare. Forme simili esistono in altre lingue: inglese and the like, or something e sono state denominate general extenders (Overstreet 1999) o vague categories identifiers (Channel I994).

La costruzione è caratterizzata da alcune proprietà distribuzionali: è preceduta di norma da un solo costituente, occorre in posizione finale di proposizione, è pronunciata con intonazione discendente, resa nello scritto col punto, con la virgola, i contesti in cui compare sono di solito contesti irreali che designano stati non fattuali, ipotesi, istruzioni, eventi abituali.

Le sue proprietà semantiche sono: la referenza indefinita, una restrizione di somiglianza sui referenti possibili (per cui il parlante si riferisce a una serie di elementi che includono la proposizione [XP/clause piuttosto che] ) e una componente connettiva di tipo disgiuntivo "o" (cfr. Overstreet I 999: 4 sui disjunctive general extenders: or something (like that), or whatever in cui il connettore or è esplicitato).

(35) Se vuoi la cucina bella, [della Salvarani piuttosto che], te la devi pagare! (conversazione tra amiche)

(36) Io cerco sempre angoli speciali, [un balcone o un tetto piuttosto che], ma anche, un piccolo davanzale. (http://www.forumtime.it/) 
Le proprietà distribuzionali e semantiche individuate consentono secondo le autrici di questo studio di identificare una funzione grammaticale specifica ben distinta dal piuttosto che comparativo denominata restricted indefiniteness.

$\grave{E}$ stato anche preso in considerazione lo sviluppo diacronico di piuttosto che allo scopo di accertare come sono nati i valori più recenti di congiunzione disgiuntiva e di indefinito restrittivo. Anche in questo caso è stato adottato un modello a stadi, che è compatibile con l'idea di grammaticalizzazione come processo complesso in cui interagiscono mutamenti semantici e strutturali.

I. stadio: piuttosto che (spesso nei testi antichi anche più tosto che) ha in origine valore temporale, concreto "più rapidamente che", che presto sviluppa un valore astratto di preferenza tra due elementi comparati. La priorità temporale suggerisce infatti un'inferenza di preferibilità: ciò che viene prima è interpretato come preferibile, migliore.

2. stadio: nell'Ottocento piuttosto che comincia a comparire insieme a elementi non specifici, indefiniti "uno o l'altro" in cui due alternative sono presentate come intercambiabili: "una proprietà o l'altra"; la preferenza di $a$ su $b$ è equivalente alla preferenza di $b$ su $a$. Sono questi i contesti critici in cui il mutamento semantico da comparativo a congiunzione disgiuntiva si sviluppa:

(37) non potendo noi discorrere in nessun modo della natura di quegli esseri che non possiamo concepire; e non avendo nessun possibile fondamento per attribuire ad un essere posto fuori della materia, una proprietà piuttosto che un'altra, una maniera di esistere, la semplicità o la composizione, l'incorruttibilità o la corruttibilità.

(Giacomo Leopardi, Lo Zibaldone, 4. Feb. I82 I)

(38) E è dunque dimostrato, anche col fatto, che ogni effetto grammaticale può essere ottenuto con mezzi diversi; e che, per conseguenza, l'applicazione d'uno piuttosto che d'un altro di essi, dipende da un arbitrio.

(Alessandro Manzoni, Della lingua italiana. Quinta redazione e appendice)

3. stadio: una relazione comparativa preferenziale tra due esemplari presi a caso in un set più ampio è logicamente equivalente a una relazione disgiuntiva tra esemplari presi a caso in una lista non esaustiva: piuttosto che assume il valore indefinito disgiuntivo, che oggi si è parecchio diffuso anche nella prosa giornalistica, anche se l'uso viene talora biasimato (De Santis 200I). 
(39) c'è il vantaggio che ti puoi customizzare la macchina come vuoi, in relazione alle tue esigenze (grafica, piuttosto che sviluppo, piuttosto che giochi...)

(dialogo sulle caratteristiche del desktop)

4. stadio: piuttosto che con valore di connettivo disgiuntivo introduce espressioni indefinite come altre cose di questo genere (es. 40), ossia costruzioni indefinite con una restrizione di somiglianza esplicita $d i$ questo genere:

(40) sfrondando concezioni personalistiche della cosa (che so, i potenti della Terra che si riuniscono ogni tanto, piuttosto che la razza aliena dedita alla distruzione della civiltà umana, [piuttosto che altre cose del genere]) [...]

5. stadio: quando le alternative potenziali sono omesse e sostituite dall'intonazione sospensiva si produce una costruzione ellittica in cui il connettivo disgiuntivo indefinito mantiene il riferimento ad alternative potenziali non specifiche.

(4I) non voglio giudicare la tua scelta di patteggiare piuttosto che!! ma hai scritto una cosa importante: "poter chiarire e ridimensionare alcune cose" (http://www.stalking.it/?p=I 556 )

Piuttosto che arriva ad acquisire una funzione referenziale indefinita.

Dunque anche la costruzione piuttosto che può essere connessa alla famiglia di costruzioni che danno voce alla vaghezza, in cui i parlanti lasciano all'ascoltatore la scelta di completare il percorso: essa ha tratti di somiglianza con il caso di tipo, anche se tipo è certamente più versatile. Un elemento che accomuna i due percorsi è anche il fatto che siano i contesti dialogici quelli in cui si sviluppano entrambi.

Come hanno mostrato gli studi sul mutamento linguistico in prospettiva costruzionista, la variazione contestuale e sociale può essere di grande ampiezza nelle costruzioni emergenti: piuttosto che come espressione di indefinitezza non è accettato da tutti, alcuni parlanti affermano addirittura di non conoscerlo. Anche qui i concetti di gradience e di gradualità aiutano a vedere meglio i percorsi di mutamento.

\section{Note}

I. Abbiamo cercato di costruire per ciascun secolo un campione il più bilanciato possibile, che includesse testi poetici, narrativi, argomentativi, tecnici, lettere private e anche un numero comparabile di parole (min 3 I I.649 - max 540.022). I testi sono stati immagazzinati per mezzo di Wordsmith Tools, 
riportati in fogli Excel e analizzati in base a parametri semantici e sintattici rilevanti.

2. L'indagine è basata su un corpus di italiano parlato, con esempi colti al volo da conversazioni, da trasmissioni televisive e da ricerche in rete su blog, forum discussions, chat lines e sul corpus NUNC http://www.corpora.unito.it/ index_nunc.php.

È stato consultato anche il LIP e il Corpus e lessico di Frequenza dell'Italiano Scritto Contemporaneo (COLFIS). Per l'indagine diacronica sono stati consultati i corpora dell'OVI, LIZ, Biblioteca Italiana.

\section{Riferimenti}

Aarts, Bas. 2007. Syntactic Gradience. Oxford: Oxford University Press.

Bazzanella, Carla \& Mirella Cristofoli. I998. "Piuttosto che e le alternative non preferenziali: un mutamento in atto?". Cuadernos de filologia italiana, 5. 267-278.

Bergs, Alexander \& Gabriele Diewald. 2008. Constructions and Language Change. Berlin/New York: Mouton de Gruyter.

Bisang, Walter, Nikolaus P. Himmelmann \& Björn Wiemer (eds). 2004. What makes Grammaticalization? A Look from its Fringes and its Components. Berlin/New York: Mouton de Gruyter.

Brucale, Luisa. 20I 2. "L'uso non canonico di 'piuttosto che' coordinativo in italiano contemporaneo". In: Bianchi, Patricia, Nicola De Blasi, Chiara De Caprio \& Francesco Montuori, La variazione nell'italiano e nella sua storia. Atti dell'XI Congresso SILFI. Firenze: Franco Cesati, 483-493.

Bybee, Joan. 2006. "From usage to grammar: the mind's response to repetition". Language, 82. 7 I I-733.

Bybee, Joan. 2007. Frequency of use and the organization of language. Oxford: Oxford University Press.

Channell, Joanna. 1994. Vague Language. Oxford: Oxford University Press.

Cristofaro, Sonia. 2005. Subordination. Oxford: Oxford University Press.

Croft, William. 2003. Typology and Universals. 2nd edition. Cambridge: Cambridge University Press.

Croft, William. 2006. Typology. The Handbook of Linguistics. Oxford: Blackwell. 337-368.

Croft William. 20I0. "The origins of grammaticalization in the verbalization of experience". Linguistics, 48:I. I-48. 
Croft William \& Alan D. Cruse. 2004. Cognitive Linguistics. Cambridge: Cambridge University Press.

Davidse, Kristin, Lieven Vandelanotte \& Hubert Cuyckens. 2010. Subjectification, Intersubjectification and Grammaticalization. Berlin: Mouton de Gruyter.

Denison, David. 2005. "The grammaticalization of sort of, kind of and type of in English". Paper presented at New Reflections on Grammaticalization 3, University of Santiago de Compostela, July I7-20, 2005.

De Santis, Cristiana. 200I. "L'uso di piuttosto che con valore disgiuntivo". Studi di Grammatica Italiana, XX. 339-350.

Diewald, Gabriele. 2002. "A model for relevant types of contexts in grammaticalization”. In: Wischer, Ilse \& Gabriele Diewald (eds.), New Reflections on Grammaticalization. Amsterdam: Benjamins.103-I20.

Diewald, Gabriele. 2006. "Discourse particles and modal particles as grammatical elements”. In: Fischer, Kerstin (ed.). 2006. Approaches to discourse particles. Amsterdam: Elsevier. 403-425.

Diewald, Gabriele. 20го. "On some problem areas in grammaticalization studies”. In: Stathi, Katerina, Elke Gehweiler \& Ekkehard König (eds.). I7-50.

Fischer, Kerstin (ed.). 2006. Approaches to discourse particles. Amsterdam: Elsevier.

Fischer, Olga. 2007. Morphosyntactic Change. Formal and Functional Perspectives. Oxford: Oxford University Press.

Fraser, Bruce. 2007. "The English contrastive discourse marker 'instead'." In: Butler, Christopher S., Raquel Hidalgo Downing \& Julia Lavid (eds.), Functional Perspectives on Grammar and Discourse. In honour of Angela Downing. Madrid: University of Wales, Swansea/Universidad Complutense. $30 \mathrm{I}-3$ I 2 .

Giacalone Ramat, Anna \& Caterina Mauri. 2008. "From cause to contrast. A study in semantic change". In: Verhoeven Elisabeth, Stavros Skopeteas, Yong-Min Shin, Yoko Nishina \& Johannes Helmbrecht (eds.). Studies on Grammaticalization. Berlin/New York: Mouton de Gruyter. 303-321.

Giacalone Ramat, Anna \& Caterina Mauri. 2009. "Dalla continuità temporale al contrasto: la grammaticalizzazione di tuttavia come connettivo avversativo". In: Ferrari, Angela (a cura di), Sintassi storica e diacronica dell'italiano: Subordinazione, coordinazione, giustapposizione. Atti del X Convegno della Società Internazionale di Linguistica e Filologia Italiana (Basilea, 30 giugno-3 luglio 2008). Firenze: Franco Cesati Editore. 449-470. 
Giacalone Ramat, Anna \& Catherine Camugli Gallardo. 20 I . "L'emploi des connecteurs: però correspond-il toujours à mais ?”. Revue Française de linguistique appliquée, XVI:2. 57-74.

Giacalone Ramat, Anna, Caterina Mauri \& Piera Molinelli. 20I3. Synchrony and Diachrony: a Dynamic Interface. Amsterdam: John Benjamins.

Goldberg, Adele E. I995. Constructions: A Construction Grammar Approach to Argument Structure. Chicago: University of Chicago Press.

Goldberg, Adele E. 2006. Constructions at work. The Nature of Generalization in Language. Oxford: Oxford University Press.

Haspelmath, Martin. 2004. "On directionality in language change with particular reference to grammaticalization”. In: Fischer, Olga, Muriel Norde \& Harry Perridon (eds.), Up and Down the Cline - the Nature of Grammaticalization [Typological Studies in Language 59]. Amsterdam: John Benjamins. I7-44.

Heine, Bernd. 2002. "On the role of context in grammaticalization". In: Wisher, Ilse \& Gabriele Diewald (eds.). New Reflections on Grammaticalization. Amsterdam/ New York: John Benjamins. 83-IOI.

Hopper, Paul \& Elisabeth C. Traugott. 2003. Grammaticalization. 2nd ed. Cambridge: Cambridge University Press

Labov, William. I972. Sociolinguistic Patterns. Philadelphia: University of Pennsylvania Press.

Labov, William.I994. Principles of Linguistic Change: Internal factors. Oxford: Blackwell.

Labov, William. 200I. Principles of Linguistic Change: Social factors. Oxford: Blackwell.

Lehmann, Christian. I982. “Thoughts on grammaticalization: a Programmatic Sketch". In: Arbeiten des Kölner Universalien Projektes, Nr. 48. Köln: Institut für Sprachwissenschaft. Ripubblicato: München, LINCOM EUROPA, I 995.

Lehmann, Christian. 2005. "Theory and method in grammaticalization". Zeitschrift für Germanistik und Linguistik, 32:2, 2004. I 52-I 87.

Margerie, Hélène. 20ıо. "On the rise of (inter)subjective meaning in the grammaticalization of kind oflkinda. Subjectification, intersubjectification and grammaticalization". In: Davidse, Kristin, Lieven Vandelanotte \& Hubert Cuyckens (eds.). 2010. Subjectification, Intersubjectification and Grammaticalization. Berlin: De Gruyter Mouton. 3 I 5-346.

Mauri, Caterina \& Anna Giacalone Ramat. 20I I. "Restricted indefiniteness: the case of Italian piuttosto che". (Paper presented at the SLE 44th Annual Meeting -Logroño, 9-I I September 20II). 
Mauri, Caterina \& Anna Giacalone Ramat. 20I 2. "The development of Italian adversative connectives: factors at play and stages of grammaticalization”. Linguistics, 50:2. I9I-239.

Martinet, André.r955. Economie des changements phonétiques. Berne: Francke.

Martinet, André. 1960. Eléments de linguistique générale. Paris: Armand Colin.

Mihatsch, Wiltrud. 2007. "The construction of vagueness: sort of expressions in Romance languages”. In: Radden, Günter, Klaus-Michael Köpke, Thomas Berg \& Peter Siemund (eds.). Aspects of meaning constructing meaning: From concepts to utterance. Amsterdam/Philadelphia: John Benjamins. 225-245.

Overstreet, Marianne. I999. Whales, Candlelight, and Stuff Like That: General Extenders in English Discourse. New York: Oxford University Press.

Saussure, Ferdinand de. I955 [I916]. Cours de Linguistique Générale. 5 éd. Paris: Payot.

Scorretti, Mauro. I988. "Le strutture coordinate”. In: Renzi, Lorenzo (ed.). Grande grammatica italiana di consultazione, vol. I. Bologna: Il Mulino. 227-270.

Serianni, Luca. I989 (con la collaborazione di Alberto Castelvecchi). Grammatica italiana. Italiano comune e lingua letteraria. Torino: UTET Libreria.

Stathi, Katerina, Elke Gehweiler \& Ekkehard König. 20 Io. Grammaticalization. Current views and issues. Amsterdam/Philadelphia: John Benjamins.

Traugott, Elizabeth C. I989. "On the rise of epistemic meanings in English: an example of subjectification in semantic change”. Language, 57.33-65.

Traugott, Elisabeth C. 2003. "Constructions in Grammaticalization". In: Joseph, Brian D. \& Richard D. Janda (eds.). A Handbook of Historical Linguistics. Oxford: Blackwell. 624-647.

Traugott, Elisabeth C. 2008. "The grammaticalization of NP of NP constructions”. In: Bergs, Alexander \& Gabriele Diewald (eds.). Constructions and Language Change. Berlin/New York: Mouton de Gruyter. 2 I-43.

Traugott, Elizabeth C. 20Iо. "(Inter)subjectivity and (Inter)subjectification”. In: Davidse, Kristin, Lieven Vandelanotte \& Hubert Cuyckens (eds.). Subjectification, intersubjectification and grammaticalization. Berlin/New York: Mouton de Gruyter. 29-7I.

Traugott, Elisabeth C. \& Ekkehard König. I99I. "The pragmatics of grammaticalization revisited". In: Traugott, Elisabeth C \& Bernd Heine (eds.). Approaches to grammaticalization. Vol. I: Focus on theoretical and methodological issues. Amsterdam: John Benjamins. I 89-2 I 8. 
Traugott, Elisabeth C. \& Richard B. Dasher. 2002. Regularity in semantic change. Cambridge: Cambridge University Press.

Traugott, Elisabeth C. \& Graeme Trousdale. 2010. Gradience, Gradualness and Grammaticalization. Amsterdam: John Benjamins.

Trousdale, Graeme. 20I0. "Issues in constructional approaches to grammaticalization in English". In: Stathi, Katerina, Elke Gehweiler \& Ekkehard König (eds.). Grammaticalization. Current views and issues. Amsterdam/ Philadelphia: Benjamins. 5 I-7I.

Trousdale, Graeme 20I3. "Gradualness in language change: a constructional perspective”. In: Giacalone Ramat, Anna, Caterina Mauri \& Piera Molinelli (eds.). Synchrony and Diachrony: a Dynamic Interface. Amsterdam: John Benjamins. 27-42.

Van Gelderen, Elly. 20I0. "Features in reanalysis and grammaticalization". In: Traugott, Elisabeth C. \& Graeme Trousdale (eds.). Gradience, gradualness and grammaticalization. Amsterdam: John Benjamins. I 29-I 47.

Van Linden, An, Jean-Christophe Verstraete \& Kristin Davidse. 2010. Formal Evidence in Grammaticalization Research. Amsterdam: Benjamins.

Voghera, Miriam. 20I 2. "When vagueness implies (categorization by) similarity". (Paper presented at the $45^{\text {th }}$ Annual Meeting of the Societas Linguistica Europaea, Stockholm 29 August - I September 20I2).

Voghera, Miriam. 20I3. "A case study on the relationship between grammatical change and synchronic variation: the emergence of tipo $_{[-\mathrm{N}]}$ in Italian”. In: Giacalone Ramat, Anna, Caterina Mauri \& Piera Molinelli (eds.). Synchrony and Diachrony: a dynamic interface. Amsterdam: John Benjamins. 283-3 I I.

Waltereit, Richard. 2002. "Imperatives, interruption in conversation, and the rise of discourse markers: a study of Italian guarda". Linguistics, 40. 987-IOIO.

Waltereit, Richard. 2006. "The rise of discourse markers in Italian: a specific type of language change". In: Fischer, Kerstin (ed.). Approaches to discourse particles. Oxford: Elsevier. 6I-76. 